\title{
Is there a role for adjuvant therapy for resected non-small cell lung cancer?
}

\author{
James R Jett \\ Department of Pulmonary Medicine and Medical Oncology, Mayo Clinic, \\ Rochester, Minnesota 55905, USA
}

Introductory article

\section{Postoperative radiotherapy in non-small-cell lung cancer: systematic review and meta-analysis of individual patient data from nine randomised controlled trials}

\author{
PORT Meta-Analysis Trialists Group
}

Background. The role of postoperative radiotherapy in treatment of patients with completely resected non-small-cell lung cancer (NSCLC) remains unclear. We undertook a systematic review and meta-analysis of the available evidence from randomised trials. Methods. Updated data were obtained on individual patients from all available randomised trials of postoperative radiotherapy versus surgery alone. Data on 2128 patients from nine randomised trials (published and unpublished) were analysed by intention to treat. There were 707 deaths among 1058 patients assigned postoperative radiotherapy and 661 among 1072 assigned surgery alone. Median follow-up was 3.9 years (2.3-9.8 for individual trials) for surviving patients. Findings. The results show a significant adverse effect of postoperative radiotherapy on survival (hazard ratio 1.21 [95\% Cl 1.08-1.34]). This 21\% relative increase in the risk of death is $\mathbb{Q}$ equivalent to an absolute detriment of $7 \%$ (3 11) at 2 years, reducing overall survival from $55 \%$ to $\overrightarrow{0}$ $48 \%$. Subgroup analyses suggest that this adverse effect was greatest for patients with stage IIII NO-N1 disease, whereas for those with stage III N2 disease there was no clear evidence of an adverse effect. Interpretation. Postoperative radiotherapy is detrimental to patients with early-stage completely resected NSCLC and should not be used routinely for such patients. The role of postoperative radiotherapy in the treatment of N2 tumours is not clear and may warrant further research (Lancet 1998;352: 257-63)

\begin{abstract}
Meta-analysis was developed as a technique to summarise the results of multiple related studies. A good meta-analysis requires a systematic approach to the identification and abstracting of critical information from each report being analysed. Investigators must identify all relevant studies by carefully reviewing data bases and bibliographies and by seeking out unpublished reports. This technique allows inclusion of small studies and both published and unpublished trials. Unpublished trials are included in an effort to avoid publication bias. Ideally, reports in other languages are included. Proper use of this methodology necessitates extensive collaboration between clinicians and statisticians. When performed correctly, meta-analysis should provide us with the "best estimate" of the answer to the question under evaluation.

Large randomised prospective clinical trials are considered the "gold standard" and are assigned the highest level of evidence when developing clinical guidelines. Should the results of meta-analysis displace results from large randomised controlled trials? LeLorier and colleagues compared the results of large randomised control
\end{abstract}

trials (1000 patients or more) with the results of metaanalyses published earlier on the same topics. They of identified 12 large randomised controlled trials and $N$ 19 meta-analyses addressing the same questions. The 요 agreement between the meta-analyses and the large $\omega$ trials was only fair $($ kappa $=0.35)$. The positive predictive value (PPV) of meta-analysis was $68 \%$ and the negative predictive value (NPV) was $67 \%$. Accordingly, $\stackrel{?}{?}$ if there had been no subsequent randomised trial, the meta-analysis would have led to the adoption of in- 0 effective treatment in $32 \%$ of cases $(100 \%$ minus the $\stackrel{\mathbb{Q}}{\stackrel{Q}{Q}}$ PPV) and to rejection of a useful treatment in $33 \%$ of $\underset{\mathbb{8}}{\mathbb{8}}$ cases $(100 \%$ minus the NPV). In all cases of disagreement a statistically significant effect of treatment was found by either the meta-analysis or the larger 0 randomised trial and no significant effect by the other method. In no case was there a divergence in which the large randomised trial and the meta-analysis gave statistically significant and opposite answers - that is, a positive effect to a detrimental effect. The report by LeLorier et of should certainly make us think twice before uncritically accepting the result of the next metaanalysis we read. 


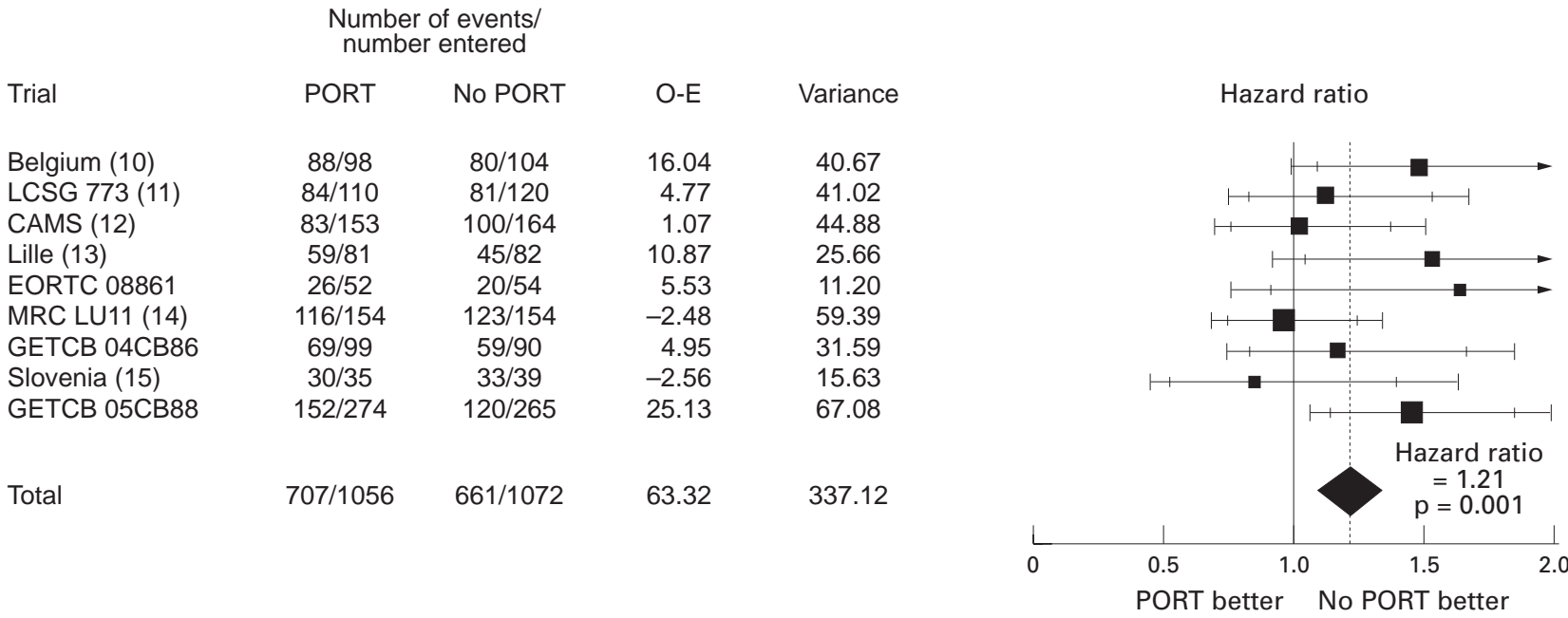

Figure 1 Hazard ratio plot for survival. Each individual trial is represented by a square, the centre of which denotes the hazard ratio for that trial: extremities of horizontal bars denote $99 \% \mathrm{Cl}$ and inner bars mark $95 \% \mathrm{Cl}$. The size of the square is directly proportional to the amount of information in the trial. The black diamond at the foot of the plot gives the overall hazard ratio for combined results of all trials; the centre denotes the hazard ratio and extremities $95 \% \mathrm{Cl}$. Trials are ordered chronologically by date of start of trial (oldest first). PORT=postoperative radiotherapy. Overall hazard ratio=1.21 (95\% CI 1.08 to 1.34); $\chi_{1}{ }^{2}=11.892, p=0.001$; heterogeneity $\chi_{2}{ }^{2}=13.067, p=0.11$. Reproduced from reference 3 with permission. Numbers in parentheses refer to references in original article.

\section{Strengths and weaknesses of the postoperative radiotherapy (PORT) meta-analysis}

In the meta-analysis discussed in the Introductory Articl $3^{3}$ the authors conclude that postoperative radiotherapy (PORT) in patients with completely resected non-small cell lung cancer had a statistically significant adverse effect on survival (hazard ratio (HR) 1.21 (95\% CI 1.08 to 1.34 )) and reduced overall survival at two years from $55 \%$ to $48 \%$ (figs 1 and 2 ).

What are the strengths of the PORT meta-analysis? The study was conducted by an outstanding group of investigators with a well established track record in this type of analysis. They performed a comprehensive literature review and included both published and unpublished trials. They used updated information on survival, recurrence, date of last follow up, details of treatment allocation, randomisation, age, sex, cell type, stage, and performance scores. Information was obtained for all randomised patients including those who had been excluded from the investigator's original analyses. All trials included were analysed on an intention

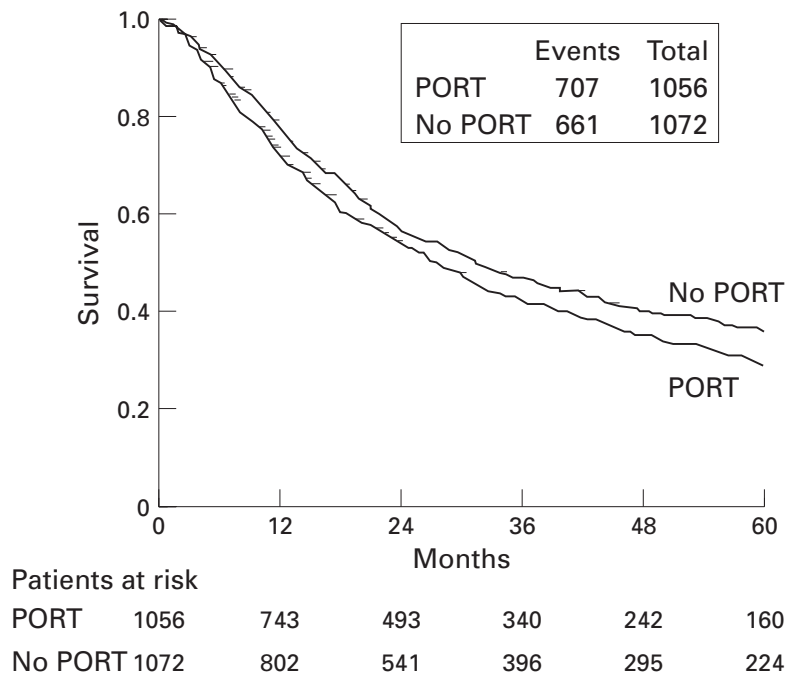

Figure 2 Kaplan-Meier curve for survival. Reproduced from reference 3 with permission. to treat basis. In addition to the main conclusion that postoperative radiotherapy was detrimental, no subgroup analysis based on age, sex, or histology dem- $\vec{\bullet}$ onstrated a benefit with treatment. Additionally, analysis based on disease stage did not show a beneficial effect of PORT in patients with stage I, II, or III.

Are there any weaknesses? Definitely yes! The analysis includes studies that were performed over a 30 year period (1965-95). The total dose of radiotherapy varied $\stackrel{\mathbb{Q}}{2}$ from 30 to $60 \mathrm{~Gy}$ and the number of treatment fractions $\overrightarrow{\overrightarrow{0}}$ varied from 10 to 30 . The radiation sources employed 3 varied from cobalt 60 (gamma ray teletherapy) to linear accelerator (megavoltage $x$ ray therapy). Of importance is the fact that only one trial used computed tomographic planning of radiotherapy. The differences in the number of fractions, dose per fraction, total dose, as well as $\times$ source of radiation and other quality control issues such 3 as portal size and percentage of total planned dose delivered to the port could all influence the outcomes of the individual trials.

An additional weakness of the analysis is the inclusion of patients with a performance score (PS) of 2, 3, or 4 . Of the 488 patients with a known performance score, 160 were PS 2,3 , or 4 . In current clinical trials patients $\sigma$ with a PS of 3 or 4 are almost never enrolled because $\tilde{N}$ of excessive treatment related morbidity and mortality in these poor risk individuals. Especially problematic is $\underset{2}{ }$ the fact that PS was not known for patients enrolled in 6 six of the clinical trials included in the analysis. It is $\mathbb{D}$ also unclear whether the various trials stratified treat-? ment based on PS.

Finally, 15\% of deaths in the individuals randomised to PORT were due to non-cancer and non-treatment related causes while only $9 \%$ of deaths in the surgery only group were due to these factors. Was this difference due to the chance occurrence of more cardiovascular of or other disease in the PORT group compared with the no-PORT group? Probably not, but we do not really 을 know because the trials were not controlled for comorbid conditions. This difference in non-cancer deaths in the two arms suggests that $6 \%$ of the patients in the radiotherapy group were dying even though these were not clearly established as treatment related deaths. This suggests real quality control issues related to the PORT 
and it would be interesting to know if more of these deaths due to "other causes" were observed in the earlier trials from the late 1960s and early 1970s.

\section{Review of selected articles included in the PORT meta-analysis}

The Belgium trial reported by VanHoutte and colleagues enrolled 224 patients from 1966 to 1975 All patients had a curative resection defined as "the tumour must be completely removed without extension beyond the lung and without invasion of the lymph nodes". One week after surgery patients were randomised to receive adjuvant radiotherapy (PORT) or no PORT. The radiation source was a cobalt 60 unit and the dose was 6000 cGy in 30 fractions. Only 175 patients of the initial 224 were evaluable. Radiation treatment was not performed in 12 patients randomised to radiotherapy. The five year survival was lower in the PORT group $(24 \%$ vs. $43 \%)$ but the difference was not statistically significant.

By current standards the limitations of the Belgium trial were that it covered a nine year period in the late 1960 s and early 1970s and used a cobalt 60 machine as the source of irradiation. Simulator planning was not used because this was not generally available until the mid 1970s. Furthermore, this trial preceded CT planning of radiotherapy fields which became available in the late 1970s. The staging system employed was the 1977 American Joint Committee for Staging and End Result Reporting. Also problematic was the fact that 224 patients were admitted to the trial but only 175 were considered evaluable. It is unclear whether the 12 patients randomised to radiotherapy but who did not receive treatment were included in the analysis. While this trial was superb for its time, there are numerous problems with design, implementation, and analysis based on the standards of the 1990s.

The Lung Cancer Study Group randomised 230 patients with stage II or III totally resected squamous cell cancer to observation or PORT from 1978 to 1985 . A total of $50 \mathrm{~Gy}$ was delivered in $1.8-2.0 \mathrm{~Gy}$ daily fractions. The radiation sources were either cobalt 60 or linear accelerators. Of 230 patients randomised, 210 were eligible. Ineligibility (17 of 20 ) was due mainly to non-squamous histology at the time of pathological review. Of 210 patients, 10 did not receive the assigned radiotherapy and three of those assigned to no radiotherapy demanded treatment. The time to local tumour recurrence favoured the PORT arm but was not statistically significant $(\mathrm{p}=0.188)$. The overall survival of the two groups was identical with overlapping curves (fig 3). The number of non-cancer deaths in the PORT arm included six from respiratory failure and five from cardiac failure while in the control arm there were three deaths from respiratory failure and two from cardiac failure. Subset analysis, with all its potential problems, suggested a reduced local recurrence rate in patients with resected N2 disease; however, no survival benefit was observed with radiotherapy in any nodal disease subgroup.

This trial had an excellent design and careful analysis with a less than $10 \%$ ineligibility rate. Two potential weaknesses of the trial were that it took seven years to complete and included only squamous cell histology, thereby raising the question of how applicable it is to other cell types. To date, we do not have clear evidence that the various histological cell types of non-small cell lung cancer should be treated differently. The results of this trial are convincing in that PORT does not

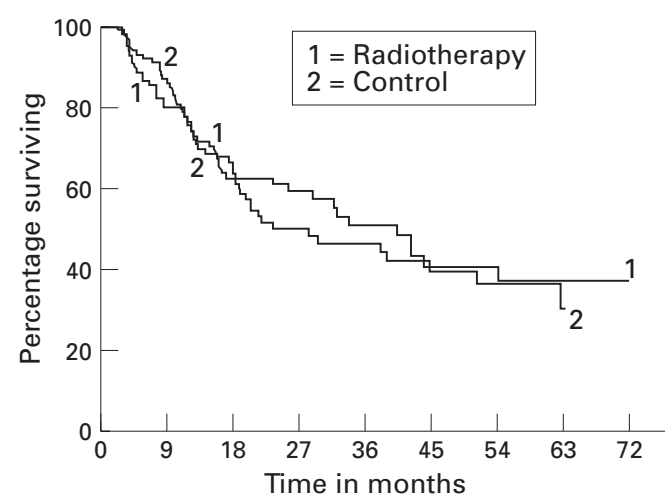

No. at risk

Radiotherapy

Control

$\begin{array}{lll}51 & 23 & 12 \\ 48 & 23 & 10\end{array}$

Figure 3 Time to death (from any cause) according to study group. The difference between the groups was not significant $(p=0.678$, log rank test). Reproduced from reference 5 with permission.

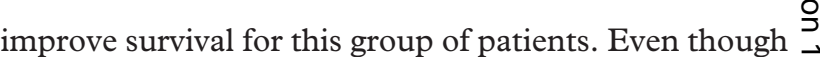
CT planning of radiation ports was not employed, there $\gtrsim$ were only a few excess deaths that could be considered treatment related in the PORT arm. With today's tech- $\stackrel{\infty}{\oplus}$ nologies of CT planning and dosimetry measurement $\vec{\bullet}$ a treatment related death should be very rare, especially $\mathbb{\&}$ in the adjuvant setting.

The Medical Research Council conducted a 16 centre trial in the United Kingdom from 1986 to 1993 that included 308 patients 6 Patients were carefully staged as T1N1M0, T2N1M0, T1N2M0, and T2N2M0. They were stratified by surgeon, TNM stage, and histology. The radiation dose was $40 \mathrm{~Gy}$ in 15 fractions using either cobalt 60 or linear accelerator. At the time of the report $72 \%$ of the 308 patients had died. The survival curves were overlapping with a hazard ratio of $1.00(\mathrm{p}=$ 0.99; fig 4). Overall, the time to local recurrence did $\underline{0}$ not differ between the two arms (HR 1.23, 95\% CI 0.87 to $1.73, \mathrm{p}=0.24)$. In subgroup analysis the $\mathrm{N} 2 \stackrel{\mathrm{\sigma}}{7}$ group receiving PORT appeared to have a delayed time to local recurrence.

This trial was well designed, conducted, and analysed. The PORT group did have treatment related toxicity, 은 including 15 classified as severe, but there were no $\rightarrow$ treatment related deaths. The authors themselves concluded that radiation toxicity was "not of sufficient $N$

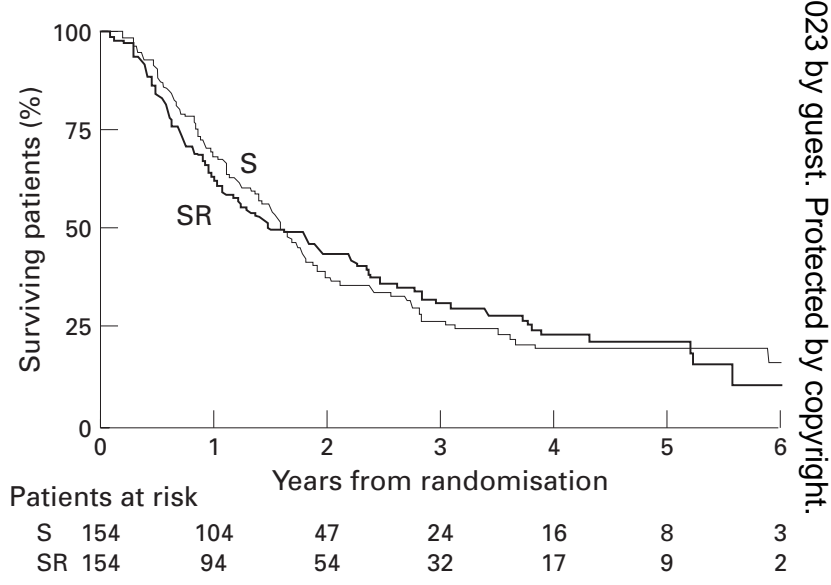

Figure 4 Percentage of patients surviving from date of randomisation. $S=$ surgery alone; $S R=$ surgery + postoperative radiotherapy. Reproduced from reference 6 with permission. 
magnitude to be regarded as a contraindication to treatment". They also point out that the radiotherapy dose and techniques used would not currently be regarded as ideal. The midline mediastinal nodes received a lower dose as a result of posterior spinal cord shielding. In the USA a dose of $60 \mathrm{~Gy}$ in 30 fractions over six weeks would be considered a more standard regimen.

Role of postoperative adjuvant chemotherapy A now classic meta-analysis was performed in collaboration between the Medical Research Council Cancer Trials Office and Institute Gustave Roussy. They evaluated trials from 1965 to the end of 1991. Patients with early stage disease underwent surgical resection and were randomised to receive systemic chemotherapy or no treatment. Eight of the more recent trials, which included almost 1400 patients, used cisplatin based combination chemotherapy and five older trials used alkylating agents (mainly cyclophosphamide and nitrosourea). The results of the trials involving surgery followed by alkylating agents were all consistent with worse survival in the adjuvant chemotherapy arms. The hazard ratio was $1.15(p=0.005)$ in favour of surgery alone. For regimens containing cisplatin the hazard ratio estimates for most trials favoured chemotherapy, with an overall hazard ratio of $0.87(\mathrm{p}=0.08)$ or a $13 \%$ reduction in the risk of death, suggesting an absolute benefit from chemotherapy of $5 \%$ at five years.

In the same meta-analysis the authors evaluated randomised trials of surgery plus radiotherapy versus surgery plus radiotherapy plus chemotherapy. The overall hazard ratio of 0.94 was marginally in favour of chemotherapy $(p=0.46)$. Collectively, these meta-analyses suggest that adjuvant chemotherapy after surgery alone or surgery and radiotherapy adds marginally to survival. In current practice, patients with totally resected stage IA/B, IIA/B, or IIIA tumours are not routinely offered either adjuvant chemotherapy or radiotherapy or the combination. Surgery alone is the standard even though the five year survival rate is helow $50 \%$ for patients with stage IIA/B or IIIA disease 8 To date, adjuvant therapy of any type has not been proved to improve survival.

At the 1999 American Society of Clinical Oncology Meeting, Keller and associates presented the results of an intergroup collaborative surgical adjuvant trial.9 Patients with totally resected stage II or IIIA non-small cell lung cancer were randomised to receive thoracic radiotherapy (50 Gy/28 fractions), with or without four cycles of concurrent chemotherapy with etoposide and cisplatin. All patients had careful mediastinal lymph node staging. It took over six years to enroll 480 patients. There was absolutely no difference in survival between patients receiving radiotherapy alone and those receiving $\times$ the combined modality therapy. When this trial was $\underset{\vec{F}}{\vec{D}}$ launched adjuvant radiotherapy was commonly employed in resected patients with $\mathrm{N} 1$ or $\mathrm{N} 2$ disease even $\frac{\mathrm{C}}{\mathrm{O}}$ though it had not been shown to improve survival. $\overline{\bar{p}}$ Accordingly, investigators did not believe that it would $\underset{\otimes}{\nabla}$ be possible to conduct a trial with a "no treatment" arm in North America. Because of the public's perception that treatment is better than no treatment, it was decided to give radiotherapy to all patients and to $\vec{\omega}$ ask the additional chemotherapy question. The answer to that question is that etoposide/cisplatin chemotherapy does not add to survival in patients with totally resected in disease. ${ }^{9}$

In North America there are currently two adjuvant chemotherapy trials underway for totally resected dis- is ease. The National Cancer Institute of Canada has an intergroup trial (BR10) that randomises patients with 은 totally resected non-small cell lung cancer (T2NOM, $\mathrm{T} 1 \mathrm{~N} 1 \mathrm{M} Q \mathrm{~T} 2 \mathrm{~N} 1)$ to receive four cycles of cisplatin $\vec{C}$ $\left(50 \mathrm{mg} / \mathrm{n}^{2}\right.$ on days 1 and 8$)$ and weekly vinorelbine (25 mg/n耳 for 16 weeks) or observation only. The goal $\stackrel{\oplus}{+}$ of the study is to compare disease free and overall $\vec{\bullet}$ survival in the two groups and to compare the quality of life for treatment versus no treatment.

A second adjuvant chemotherapy trial is being conducted by the Cancer and Acute Leukemia Group B in collaboration with other cooperative oncology groups. ٌٌ Patients with T2N0M0 non-small cell lung cancer are $\mathbb{8}$ randomised either to observation alone or to treatment $\overrightarrow{\overrightarrow{0}}$ with four cycles of systemic chemotherapy with pacli- 3 taxel $\left(200 \mathrm{mg} / \mathrm{m}^{2}\right)$ by three hour infusion and carboplatin $(\mathrm{AUC}=6)$. The end point of the trial is survival. This trial and the NCI Canada trials are currently accruing patients. It will be several years before we know the results of these trials. In Europe the Adjuvant Lung Project Italy (ALPI) trial randomises patients with 3 . totally resected stage I-IIIA non-small cell lung cancer to observation alone or to treatment with three cycles of mitomycin, vindesine, and cisplatin. Radiotherapy is left up to the discretion of the individual institution but, if given, it must be given to patients in both arms of

\section{LEARNING POINTS}

* The results of meta-analyses do not always correlate with the results of very large (more than 1000 subjects) randomised prospective clinical trials.

* Randomised prospective trials of adjuvant thoracic radiotherapy after complete resection of stages I-IIIA non-small cell lung cancer have not shown a survival advantage.

* There is no proven role for adjuvant chemotherapy for patients with totally resected stages I-IIIA non-small cell lung cancer.

* With surgery only the five year survival is $57 \%$ for patients with stage IB non-small cell lung cancer, $55 \% / 39 \%$ for those with stage IIA/B disease, and $25 \%$ for those with stage IIIA disease. These poor survival rates justify further efforts to evaluate the efficacy of new adjuvant therapies.

* No progress will be made in this difficult field without enrolling patients into randomised prospective trials. 
the study. Enrollment to this trial closed in January 1999.

\section{Neoadjuvant therapy for early stage non-small cell lung cancer}

The randomised trials of preoperative chemotherapy (neoadjuvant therapy) for potentially resectable stage IIIA disease by Roth at and Rosell et al are now classics in the literature ${ }^{1} \mid{ }_{11}$ These two trials are the best available on the effect of neoadjuvant chemotherapy and both show that preoperative chemotherapy followed by surgery is better than surgery alone for stage IIIA disease. Currently, a large North American intergroup trial is underway to evaluate both chemotherapy and radiotherapy with or without surgery for stage IIIA disease. This trial will help answer the question about the role of surgery in patients with stage IIIA disease, which is a subject of considerable controversy.

At the 1998 meeting of the American Society of Clinical Oncology investigators reported the preliminary results of a phase II trial evaluating treatment of patients with stage IB, IIA/B, and selected IIIA (T3N1 only) disease with two cycles of preoperative chemotherapy using paclitaxel (225 mg/n ${ }^{2}$ by three hour infusion) and carboplatin $(\mathrm{AUC}=6)$ followed by surgery ${ }^{12}$ At the time of their report $70 \%$ of patients (19 of 27) had responded to induction chemotherapy and 23 were completely resected without excessive toxicity. That trial has led to a phase III trial that will open this year. Patients with early clinical stage disease will be randomised to surgery alone or preoperative chemotherapy for three cycles with paclitaxel and carboplatin followed by surgery. The end points of the study will be survival and treatment related toxicity.

\section{Conclusions}

In summary, based on the trials reported to date, there is no clear advantage of additional treatment with either radiotherapy or chemotherapy or the combination for patients with totally resected stages I-IIIA non-small cell lung cancer. While the PORT meta-analysis con- cluded that adjuvant radiotherapy was detrimental to survival, I believe that this reflects problems with quality control in the early part of the PORT analysis before the routipe use of CT planning for radiotherapy became available ${ }^{3}$ Collectively, I believe that these trials fail to demonstrate any survival advantage with adjuvant $\stackrel{x}{.}$ radiotherapy for early stage disease. While the chemo- $\vec{\Rightarrow}$ therapy meta-analysis suggested a slight advantage for cisplatin based chemotherapy after surgical resection, the improvement in survival was marginal. This study $\frac{\bar{\sigma}}{\bar{D}}$ does, however, support the need for more studies to evaluate adjuvant chemotherapy, especially with new agents, for patients with stage IB, IIA/B, and IIIA totally के resected non-small cell lung cancer. Current ongoing $\overrightarrow{0}$ trials are outlined above, and it is possible that these studies may change the current recommendation of no adjuvant therapy for totally resected early stage disease.

1 Bailar III JC. The promise and problems of meta-analysis. N Engl f O

Med 1997;337:559-61.
2 LeLorier J, Gregoire G, Benhaddad A, et al. Discrepancies between Oे meta-analyses and subsequent large randomized, controlled trials. $N$ O Engl f Med 1997;337:536-42.

3 PORT Meta-Analysis Trialists Group. Postoperative radiotherapy in non-small cell lung cancer: systematic review and meta-analyses of individual patient data from nine randomised controlled trials. Lancet $\mathrm{O}$
1998;352:257-63.

4 VanHoutte P, Rocmans P, Smets P, et al. Postoperative radiation therapy $\rightarrow$ in lung cancer: a controlled trial after resection of curative design. Int f Radiat Oncol Biol Phys 1980;6:983-6.

5 Lung Cancer Study Group. Effects of postoperative mediastinal radiation on completely resected stage II and stage III epidermoid $\bar{c}$ cancer of the lung. N Engl F Med 1986;315:1377-81.

6 Medical Research Council Lung Cancer Working Party. The role of $\vec{\bullet}$ postoperative radiotherapy in non-small cell lung cancer: a multicentre randomized trial in patients with pathologically stage T1-2, N1-2, M0 disease. Br f Cancer 1996;74:632-9.

7 Non-Small Cell Lung Cancer Collaborative Group. Chemotherapy in non-small cell lung cancer: a meta-analysis using updated data on individual patients from 52 randomized clinical trials. BMF 1995;311: 899-909.

8 Mountain CF. Revisions in the international system for staging lung cancer. Chest 1997;111:1710-7.

9 Keller S, Adak S, Wagner $\mathrm{H}$, et al. Prospective randomized trial of $\mathbb{\mathbb { Q }}$ postoperative adjuvant therapy in patients with completely resected $\overline{\bar{B}}$ stages II and IIIA non-small cell lung cancer. Proc Am Soc Clin Oncol 1999;18:465a (\#1793)

10 Roth JA, Fossella F, Komaki R, et al. A randomized trial comparing perioperative chemotherapy and surgery with surgery alone in resectable stage IIIA non-small cell lung cancer. $\mathcal{F}$ Natl Cancer Inst 1994; 86:673-80.

11 Rosell R, Gomez-Codina J, Camps C, et al. A randomized trial comparing preoperative chemotherapy plus surgery with surgery alone in patients with non-small cell lung cancer. N Engl f Med 1994;330: 153-8.

12 Pisters KMW, Ginsberg RJ. Phase II trial of induction paclitaxel and carboplatin in early stage non-small lung cancer. Proc Am Soc Clin Oncol 1998;17:451a (\#1738). 